\title{
Clinical Investigation and Molecular Diagnosis of Microsporidian Enterocytozoon hepatopenaei (EHP) in Shrimps from Selangor, Malaysia
}

\author{
Shamala Marimuthu ${ }^{1}$ (D) Chandramathi Samudi Raju ${ }^{2}$ (D) Subha Bhassu ${ }^{1,3, *}$ (D) \\ ${ }^{1}$ University of Malaya, Faculty of Science, Institute of Biological Sciences, Department of Genetics and Microbiology, \\ Animal Genetics and Genome Evolutionary Laboratory (AGAGEL), 50603 Kuala Lumpur, Malaysia. \\ ${ }^{2}$ University of Malaya, Faculty of Medicine, Department of Medical Microbiology, 50603 Kuala Lumpur, Malaysia. \\ ${ }^{3}$ University of Malaya, Research Management and Innovation Complex, Centre for Research in Biotechnology for \\ Agriculture (CEBAR), Terra Aqua Laboratory, 50603 Kuala Lumpur, Malaysia.
}

\section{How to cite}

Marimuthu, S., Raju, C.S., Bhassu, S. (2021). Clinical Investigation and Molecular Diagnosis of Microsporidian Enterocytozoon hepatopenaei (EHP) in Shrimps from Selangor, Malaysia. Genetics of Aquatic Organisms 5(2), 67-75. http://doi.org/10.4194/2459-1831-v5_2_03

\section{Article History}

Received 05 March 2021

Accepted 28 May 2021

First Online 02 June 2021

\section{Corresponding Author}

Tel.: +60379675829

E-mail: subhabhassu@um.edu.my

\section{Keywords}

Enterocytozoon hepatopenaei (EHP)

18S rRNA

Phylogenetic

PCR

\begin{abstract}
In recent years, shrimp aquaculture production in Southeast Asia countries was highly infected by, microsporidian parasite, EHP. Recently, shrimps from farms located in Selangor were encountering growth retardation and it highly concerned us to carry out a clinical investigation in both farms (Location A and B). EHP infected P. monodon samples were collected from both locations. A total of 43 shrimp samples were collected and diagnosed via PCR using the 18S rRNA gene. Environmental parameters were found relatively higher than the advised values. It indicates that the farms are highly infected and toxic which had led to growth retardation and mortality. However, no significant differences were observed (except water temperatures) between the locations. The phylogenetic analysis expressed EHP detected from Malaysia formed in one clade and all the reference sequences clustered based on geographical continents (Asian-Latin America). Minimum-spanning network analysis shows that Malaysia samples Location A are genetically related with other Asian EHP samples even though the total positive rate of EHP infection was lower. However, samples from Location B were not clustered within the EHP clade and shows the highest genetic variance within Malaysia and among Asian samples. This preliminary investigation results confirmed the incidence of EHP outbreak in Malaysia.
\end{abstract}

\section{Introduction}

Malaysia is the top 15 aquaculture global producer and $8.9 \%$ contributes to the National Agricultural Domestic Product. According to the Southeast Asian Fisheries Development Centre (SEAFDEC), shrimp aquaculture plays a vital role in Malaysian and contributes to the country's economic growth. Infectious diseases outbreak being major concerns of shrimp aquaculture. Shrimp farms in Southeast Asia have been increasingly reporting the occurrence of severely retarded growth among shrimp populations and these shrimps found to be heavily infected with the microsporidian, Enterocytozoon hepatopenaei (EHP) (Tourtip et al., 2009; Sritunyalucksana et al., 2014). It is a parasite of penaeid shrimp, found in both black tiger prawns, $P$. monodon and Pacific white shrimp, $P$. vannamei. EHP is an obligatory, spore-forming parasite, that replicates within the cytoplasmic area of tubule epithelial cell of hepatopancreas by changing their biochemical parameters such as total protein, albumin, aspartate transaminase (AST), alanine transaminase 
(ALT) and alkaline phosphatase (Santhoshkumar et al., 2016). To date, there are two species discovered within the genus Enterocytozoon; EHP and E. bieneusi is being an intestinal parasite known to infect a wide range of mammals and birds (Freeman \& Sommerville, 2009).

EHP reported in the hepatopancreas (HP) of black tiger shrimp in Thailand in 2004 (Chayaburakul et al., 2004). It was later formally described as EHP in 2009 based on histological, ultrastructural, and phylogenetic data (Tourtip et al., 2009). EHP has been confirmed in Thailand (Tourtip et al., 2009), India (Rajendran et al., 2016), Vietnam (Ha et al., 2010), Brunei (Tang et al.,2015), Indonesia (Tang et al 2016; Artanto et al., 2020) and China (Liu et al., 2018). The first hepatopancreatic microsporidium reported from $P$. monodon in Malaysia in 1989. A recent outbreak of growth retardation has the warrant to find out the prevalence of EHP on the farms. Therefore, we aim to determine the presence of EHP in growth-retarded black tiger shrimp samples from two farms in Selangor (Location A \& B), Malaysia and to determine the relationship of EHP from various geographical regions. The genetic data obtained from this study will contribute to the status of Enterocytozoon sp. from Malaysia associated with the distribution of the species. This study will be a value-adding to provide information that will be preliminary to describe the epidemiological relationship of EHP as a pathogenic organism and for its control measures.

\section{Materials and Methods}

\section{Study Area and Measurement of Ecological Parameters}

Sampling was conducted randomly from 2 farms (Location A \& Location B) located at Selangor district right after the EHP outbreak. Pond water samples were collected and various physicochemical characteristics such as salinity $(\mathrm{g} / \mathrm{kg})$, water temperature $\left({ }^{\circ} \mathrm{C}\right)$, water $\mathrm{pH}$, dissolved oxygen (DO), nitrate, nitrite, and ammonia (NH3-N) were measured. API Freshwater Master Test Kit (MARS Fishcare, Chalfont, USA) was used to measure $\mathrm{pH}$, ammonia, nitrite, and nitrate; $\mathrm{pH}$ using $\mathrm{pH}$ meter (Sartorius PB-10 Basic pH meter, Sartorius AG, Göttingen, Germany) and dissolved oxygen, conductivity, salinity, and temperature measured using YSI Model 85 Handheld (YSI Incorporated, Yellow Springs, USA).

\section{Collection of Shrimps}

A total of 43 Black tiger prawns, P. monodon samples with the sign of stunted growth were collected from two ponds.

Live shrimps were washed and transported with continuous aeration to the laboratory. The shrimps were dissected to harvest hepatopancreases, muscle, stomach, intestine, eye, pleopods, gills and heart. Hepatopancreas was subjected to DNA extraction.

\section{Extraction of Hepatopancreas DNA}

Samples of hepatopancreas were used for DNA extraction. 120-150 mg pooled HP tissue was extracted using the modified conventional phenolchloroform method. The concentration and quality of the extracted DNA samples were checked using NanoDrop $^{\mathrm{TM}}$ 2000/2000c Spectrophotometer (Thermo Fisher Scientific, Waltham, USA).

\section{PCR Amplification}

The conventional PCR amplification targeting the SSU rRNA was carried out using the condition as follows: one cycle of initial denaturation at $94^{\circ} \mathrm{C}$ for $5 \mathrm{~min}$, followed by $30 \mathrm{cycles}$ of $95^{\circ} \mathrm{C}$ for $30 \mathrm{sec}, 57^{\circ} \mathrm{C}$ for $30 \mathrm{sec}$, $68^{\circ} \mathrm{C}$ for $45 \mathrm{sec}$, and a single step of final extension at $68^{\circ} \mathrm{C}$ for 5 mins. The reaction mixture consisted of $1 \mathrm{uL}$ of template DNA, $10 \mu \mathrm{m}$ of forward and reverse primer, $10 \mathrm{mM}$ of deoxynucleotide triphosphate, $1.5 \mathrm{uL}$ of 1.5 $\mathrm{mM}$ of $\mathrm{MgCl}_{2}$ and $1.25 \mathrm{U}$ of DNA Taq polymerase. The PCR products were then verified with $2 \%$ agarose gel electrophoresis, run at $80 \mathrm{~V}, 180 \mathrm{~mA}$ in $1 \mathrm{X}$ TAE running buffer for $40 \mathrm{~min}$. The gels were then visualized by ultraviolet transillumination and photographed. The gels were then purified using AxyPrep DNA Gel Extraction Kit (Axygen Biosciences, Union City, CA, USA) according to the manufacturer's instructions. Forward primer (5'-CGGTAACCTGTGGCTAAGAGT-3') and reverse primer (5'-TTCTCCCACACCAAGCATCG-3') (Giridharan, 2017) were used to amplify a partial fragment of SSU rRNA with an amplicon size of $597 \mathrm{bp}$. The amplification product of the EHP SSU rRNA positive samples was directly sequenced using a Sanger sequencing method (Applied Biosystems 3730XLGenetic Analyzer).

\section{Sequence Analysis}

Both the forward and reverse sequences obtained after the sequencing process were aligned using ClustalW multiple alignment algorithms and edited using Molecular Evolutionary Genetics Analysis version 7.0 (MEGA 7.0) (Kumar et al., 2016). Each of the gene sequences was BLAST analysed using NCBI/BLASTN program facility (http://www.ncbi.nlm.nih.gov/BLAST) for searching significant similarity with reference sequences available in Gen Bank. To assess genetic diversity among EHP isolates, 18S rRNA gene sequences of the EHP isolated from Malaysia were aligned with EHP sequence reported from different countries in the Gen Bank database. Enterocytozoon bieneusi (AY257180) was used as an outgroup to root the phylogenetic tree.

The EHP sequences were also analysed using MEGA 7.0 software to verify the estimate sequence divergences, and the pairwise analysis was calculated using the Kimura-2-Parameter (K2P) algorithm model. DNA Sequence Polymorphism Version 5.10.1 (DNASP v5) (Librado and Rozas, 2009) was used to generate 
haplotype data within EHP species. Network 5.0.0.1 (Bandelt et al., 1999) was used to generate a minimumspanning network (MSN) to illustrate the haplotype relationships. A minimum spanning network for each species was constructed, and the geographic origin of the haplotypes is indicated.

\section{Phylogenetic Analysis}

Two phylogenetic trees were built: i) distancebased neighbor-joining (NJ) tree in MEGA 7.0 calculated using Kimura-2-Parameter (K2P) algorithm model with 10000 bootstraps, and ii) Bayesian inference (BI) which was implemented via MrBayes version 3.1.2 (Huelsenbeck and Ronquist, 2001).

The best substitution model for $18 \mathrm{~S}$ rRNA sequences was selected using Modeltest version 3.7 software (Posada and Crandall, 1998) with the Akaike information criterion (AIC) and used in the Bayesian analysis. The best model for the sequences selected was the TIM with equal base frequencies, TIMef $(-\operatorname{lnL}=$ 0.0128; $A I C=6.0255)$. Metropolis-coupled Markov chain Monte Carlo (MCMC) was run with 30,000 generations, and the tree was sampled every 1000 generations. A split frequency probability $(P P)$ of 0.002042 was obtained from the run of MrBayes. The first $25 \%$ of the trees acquired in the analysis were discarded as burn-in, and a $50 \%$ majority-rule consensus for the remaining trees was constructed. The posterior probabilities $(P P)$ were summarised for each branch.

\section{Results}

\section{Ecological Parameters}

Sampling was conducted once growth retardation was observed in farms located in both locations. Salinity, $\mathrm{pH}$, ammonia, and nitrate level comparatively very high in both locations' farms compared with optimum water quality for shrimp culture, Figure $1 \&$ Table 1 . The salinity level of ponds in location $A$ was within the range of 10-15 ppm followed by 30ppm from location B. $\mathrm{pH}$ reading of location $B$ was comparatively high compared with location A, 9-9.2 to 8.2-8.8 respectively. Analysing on temperature, location $A$ and $B$ had the temperature in the range of $32.1-32.4^{\circ} \mathrm{C}$ and $27^{\circ} \mathrm{C}$, respectively. Nitrate and nitrite level was in the range of 5-10 ppm at both the location and nitrite was 1-2ppm. Dissolved oxygen was 5.5 at location $A$ and in the range of 6.2-6.5 at location B. Level of ammonia also higher in both the location, ranging from $8-9 \mathrm{ppm}$. The data of each parameter were analysed, and it has shown no significant differences between the two locations in terms of salinity, $\mathrm{pH}$, ammonia, nitrate and nitrite, $p>0.05$. Temperature between the locations was significantly different, $\mathrm{p}<0.05$.

Table 1 Average reading of ecological parameters of ponds from location A \& B

\begin{tabular}{lccc}
\hline Parameters & Location A & Location B & Optimum water quality for shrimp culture \\
\hline Salinity $(\mathrm{ppm})$ & $10.0 \pm 0.10$ & $30.0 \pm 0.00$ & $10-25$ \\
$\mathrm{pH}$ & $8.8 \pm 0.06$ & $9.2 \pm 0.10$ & $7.5-8.5$ \\
Ammonia $(\mathrm{ppm})$ & $8.0 \pm 0.10$ & $8.0 \pm 0.06$ & $<0.1$ \\
Nitrate $(\mathrm{ppm})$ & $10.0 \pm 0.05$ & $9.0 \pm 0.03$ & $<1.0$ \\
Nitrite $(\mathrm{ppm})$ & $2.0 \pm 0.02$ & $2.0 \pm 0.03$ & $5-10$ \\
Dissolved Oxygen $(\mathrm{mg} / \mathrm{L})$ & $5.6 \pm 0.21$ & $6.2 \pm 0.10$ & $>4$ \\
Temp $\left({ }^{\circ} \mathrm{C}\right)$ & $32.1 \pm 0.1$ & $27.0 \pm 0.01$ & $25-30$ \\
\hline
\end{tabular}

Table 2. Average percentages of pairwise genetic distances of EHP samples compared from various countries and outgroup ( $E$. bieneusi).

\begin{tabular}{|c|c|c|c|c|c|c|c|c|c|}
\hline Taxon & 1 & 2 & 3 & 4 & 5 & 6 & 7 & 8 & 9 \\
\hline (1) E. hepatopenaei (Malaysia) (Kuala Selangor) & - & & & & & & & & \\
\hline (2) E. hepatopenaei (Malaysia) (Sabak Bernam) & 4.9 & - & & & & & & & \\
\hline (3) E. hepatopenaei (Thailand) & 0.2 & 4.9 & - & & & & & & \\
\hline (4) E. hepatopenaei (Indonesia) & 0.2 & 4.9 & 0.0 & - & & & & & \\
\hline (5) E. hepatopenaei (Vietnam) & 0.2 & 4.9 & 0.0 & 0.0 & - & & & & \\
\hline (6) E. hepatopenaei (India) & 0.2 & 4.9 & 0.0 & 0.0 & 0.0 & - & & & \\
\hline (7) E. hepatopenaei (China) & 0.2 & 4.9 & 0.0 & 0.0 & 0.0 & 0.0 & - & & \\
\hline (8) E. hepatopenaei (Latin America) & 1.7 & 6.1 & 1.4 & 1.4 & 1.4 & 1.4 & 1.4 & - & \\
\hline (9) E. bieneusi (Outgroup) & 6.1 & 10.0 & 5.9 & 5.9 & 5.9 & 5.9 & 5.9 & 6.1 & - \\
\hline
\end{tabular}




\section{Sequence Analysis and Phylogenetic Topology}

A total of six EHP partial 18S rRNA sequences were successfully amplified from hepatopancreas of $P$. monodon. Out of 25 tested HP samples from Location A, 4 (16\%) were positive for EHP compared with 2 (11\%) out of 18 from Location $B$ pond were tested positive. The nucleotide sequences of all positive samples were deposited into Gen Bank database with accession numbers of SRR13298316, SRR13298317, SRR13298318, SRR 13298319, SRR13298320 and SRR13298320, BioProject \# PRJN687325. Analysis of nucleotide composition of EHP sequences involved in this study shows that the percentage of Guanine $(G)$ was the highest with $31.1 \%$, followed by Adenine (A) with $29.8 \%$, Thymine (T) with $21.9 \%$, and Cytosine (C) with $17.2 \%$. Overall, there were $93.4 \%$ conserved sites with the remaining of $6.6 \%$ variable sites. Parsimonyinformative sites and singleton sites showed $20 \mathrm{bp}$ (4.7\%) and 8 bp (1.9\%), respectively.

Reconstruction of the phylogenetic tree ( $\mathrm{NJ}$ and $\mathrm{BI}$ ) revealed that all the EHP samples were clustered based on geographical regions (Asian - America Latin) (Figure 2 \& 3). Phylogenetically, the trees demonstrated a mixed grouping of EHP from six Asian countries (i.e, Malaysia, Thailand, Indonesia, Vietnam, India, and China) which can be seen through the tree topologies. Samples from Location A formed in clusters together with other Asian samples, leads to the confirmation that EHP from Location A, Malaysia is genetically close and homogenous with the Asian samples. However, there is an exception for samples from Location B, Malaysia which is deviated from the main branch and formed a new cluster by itself. EHP from Latin America was also genetically distinct from samples of Asian countries where it has formed a separate clade. Essentially, the trees revealed monophyly of the EHP species concerning the outgroup, E. bieneusi.
Single-nucleotide polymorphisms (SNP) analysis conducted on the EHP 18S rRNA sequences excluding the outgroup revealed 28 segregating sites. Altogether, seven haplotypes were defined based on 40 sequences of EHP from seven different countries. There is a single shared haplotype $\left(H_{-} 1\right)$ and six unique haplotypes. A single shared haplotype with the highest samples frequencies is representing populations of EHP from Malaysia (a single individual of Location A), Thailand, Indonesia, Vietnam, India, and China. These countries are on the Asian continent. In furtherance, populations of EHP from Malaysia has more than one unique haplotype; Location $\mathrm{A}\left(\mathrm{H} \_1, \mathrm{H} \_2, \mathrm{H} \_5, \mathrm{H}\right.$ 6) $)$ and Location B ( $\left.H \_3, H \_4\right)$. A single haplotype $\left(H_{-} 7\right)$ is represented by the Latin American samples. A Minimum-spanning network (MSN) was generated with the haplotype data of EHP 18S rRNA sequences obtained to illustrate the relationships of EHP populations from various countries (Figure 4). The haplotype network illustrated the homogenise populations of EHP of Asian countries, except the population of Location $B$ with population disjunction observed.

The average percentages of pairwise genetic distances among the EHP samples from various countries are tabulated in Table 2 . The results of $0.0 \%$ pairwise distance revealed that EHP from Asian countries (i.e, Thailand, Indonesia, Vietnam, India, and China) was genetically identical to each other. EHP samples from Location $A$ pond shown $0.2 \%$ genetic variance with EHP samples from the Asian countries. Surprisingly, EHP from Location B became the focus of this study. The pairwise genetic distance of this group was the most distant from other populations of EHP (including Malaysia (Location A) and other Asian countries) which is $4.9 \%$. EHP from Latin America also showed the genetic distance of only $1.4 \%-1.7 \%$ with EHP of Asian countries including Malaysia (Location A), except for the Location B samples, which shows the

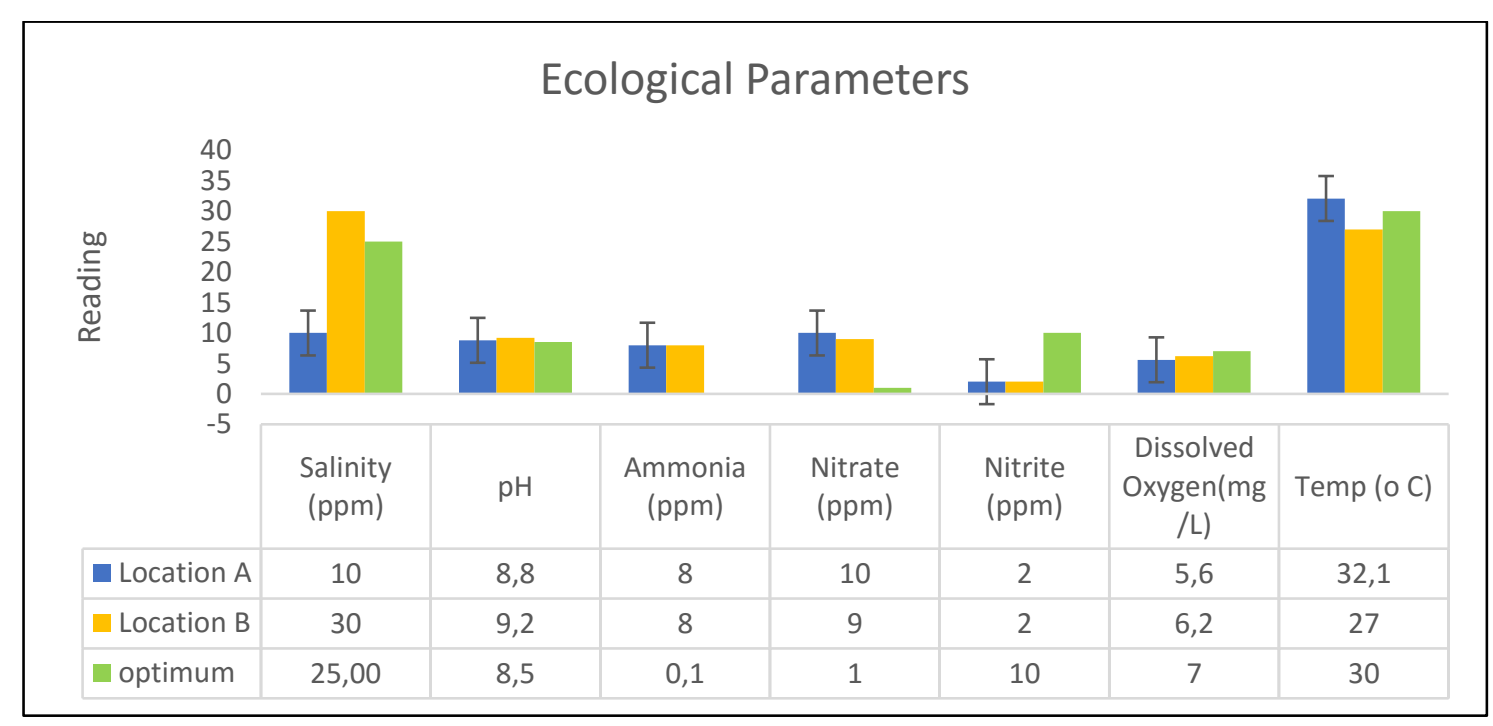

Figure 1. Average reading of ecological parameters recorded from both the locations in comparison with the optimum reading for shrimp farms. 
genetic divergence of $6.1 \%$. Populations of EHP and outgroup species of $E$. bieneusi exhibited a high value of pairwise distances, ranging from 5.9\%-10.0\% which were highly expected as they were separated in two different species.

The pairwise genetic distances between the EHP groups showed that inter-country genetic variation is higher than the intra-country relationships. From the calculation of pairwise distances, we may conclude that the genetic distances of EHP SSU rRNA sequences can explain the relationship between the species levels. This divergence analysis also supporting that samples from Location B could be a new species within Enterocytozoon genus.

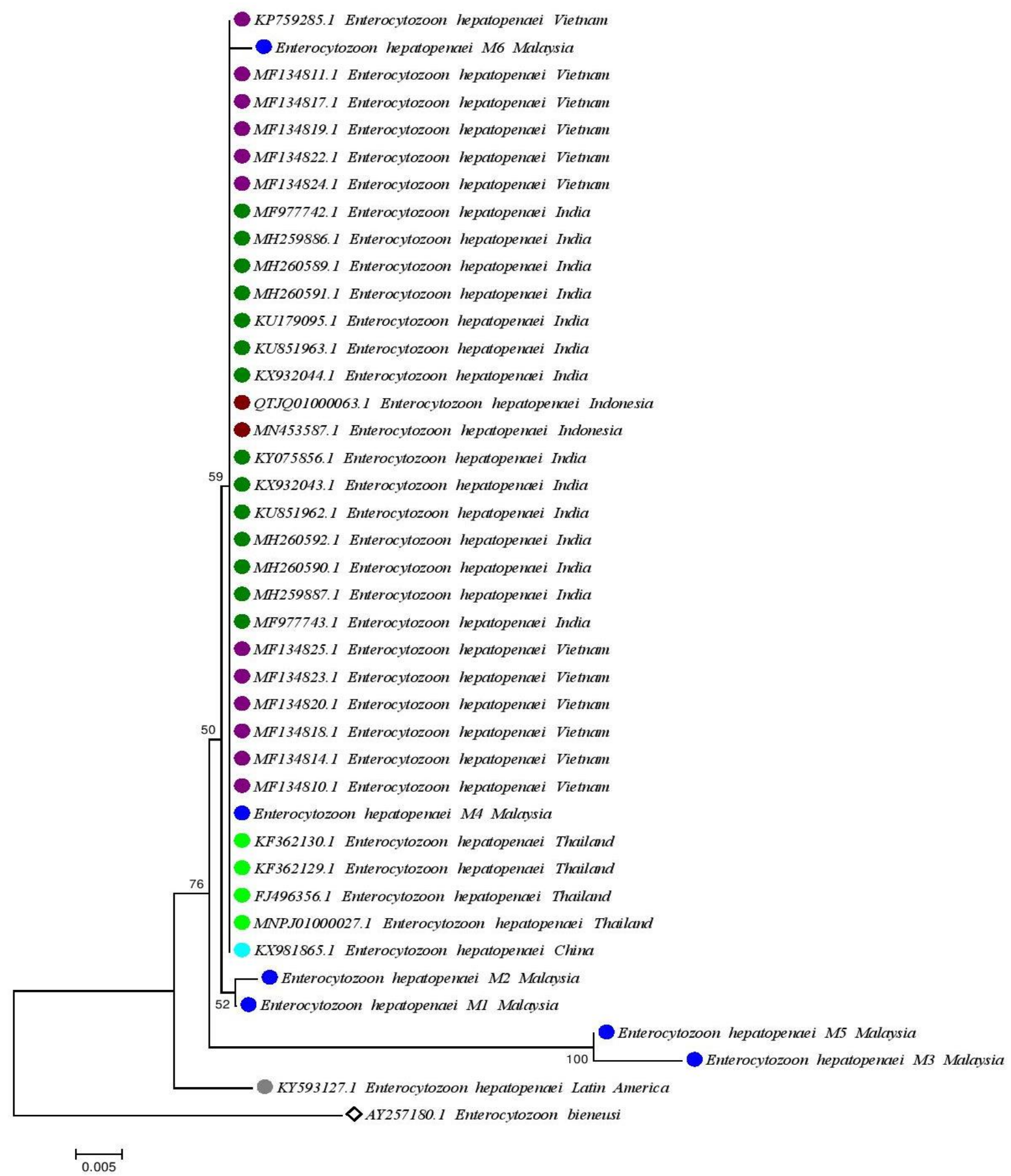

Figure 2. Neighbor- joining phylogenetic tree estimated using the Kimura-2-parameter algorithm with 10000 bootstrap replications. The optimal tree with the sum of branch length $=0.12455994$ is shown. The bootstrap values are shown next to the branches. The bar represents 0.005 nucleotide substitutions per alignment position. (All the reference sequences used in this study were EHP from P. vannamei, except EHP from P. monodon for FY496356 from Thailand, KY075856 from India and all Malaysia samples) 


\section{Discussion}

Microsporidians are a highly diverse group of spore-forming parasite infecting hosts from all major phylum in a diverse environment, especially aquatic ecosystem and cause growth retardation and lead to Hepatopancreatic Microsporidiosis. EHP mostly studies or detected in Pacific white shrimp species and spread around in Asia countries from 2002 onwards and lead to the emergence of EHP as a significant pathogen
(Thitamadee et al., 2016). EHP widely infected the Pacific white shrimp compared with black tiger shrimp, where all the reference sequences used in this study were observed from P. vannamei, except for FY496356 from Thailand and KY075856 from India which were identified from P. monodon. All Malaysia samples of EHP were also extracted from $P$. monodon. Anderson et al. (1989) reported the first outbreak of hepatopancreatic microsporidiosis in pond-reared black tiger shrimp in Malaysia.

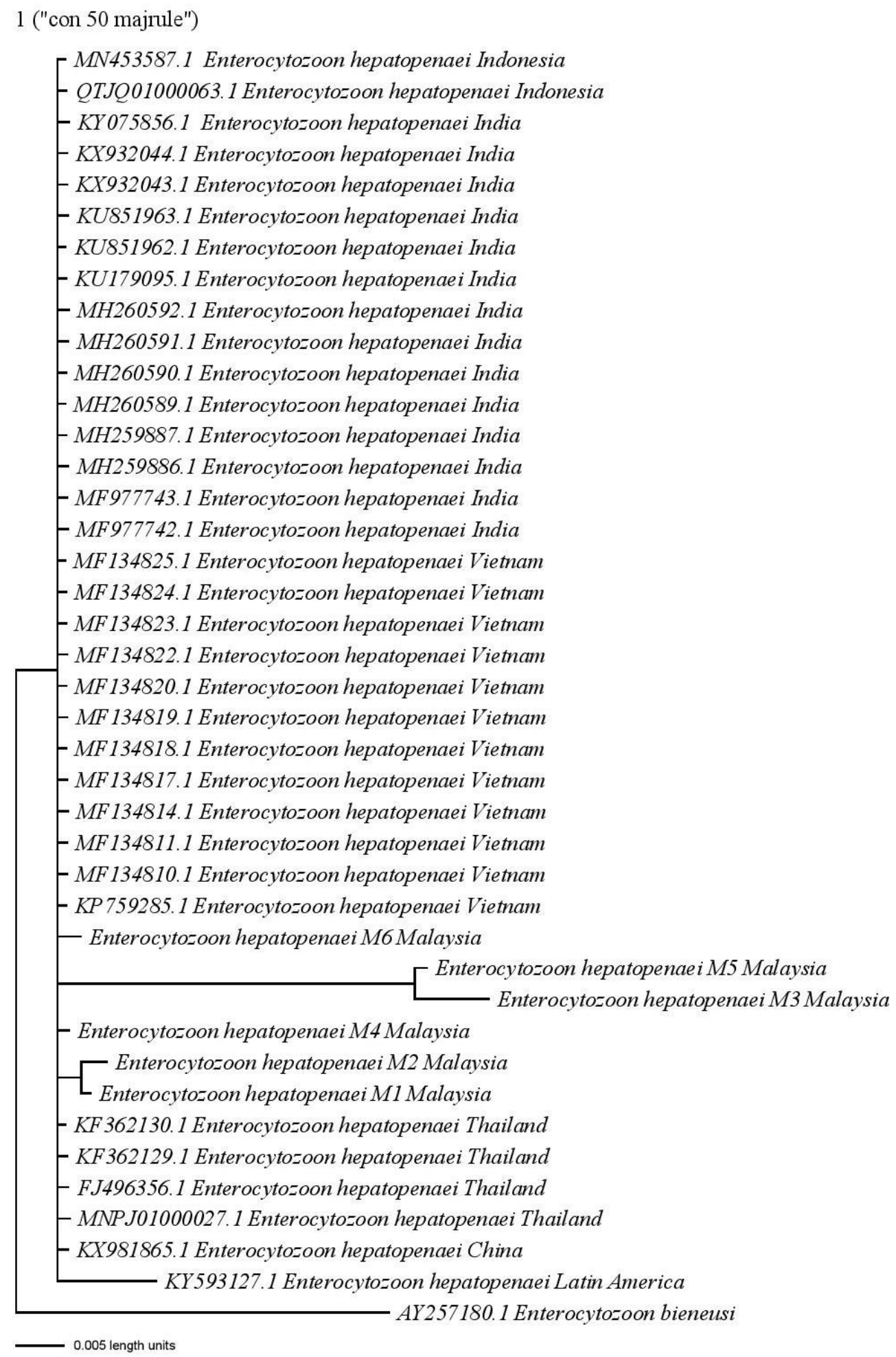

Figure 3. Bayesian inference of the EHP infected shrimps from various countries 
During sampling, gross signs include black gills, the colouration of hepatopancreas, yellow discolouration in the head and white faeces were observed. Drastic changes in water quality parameters were shown in all the investigated ponds. High ammonium level indicated as one of the stress contributing factor in shrimps. Chien (1992) reported that ammonia is toxic to shrimp at high concentration. Salinity, $\mathrm{pH}$, and dissolved oxygen levels were not within the range of acceptable shrimp culture showing the pond are highly infected too. Khal et al., (2018) \& Tangprassittipap et al., (2013) also reported that EHP infected pond's salinity is hight as 33-34 ppt and 25 ppt respectively compared with uninfected farms. The optimum temperature for the ponds ranges from 25 to $28^{\circ} \mathrm{C}$. The greater range of temperature $\left(24.5-33^{\circ} \mathrm{C}\right)$ recorded in the present study might have contributed to the slow growth and eventually mortality of the shrimps. Level of DO from both farms $>4 \mathrm{mg} / \mathrm{L}$, and it is recommended condition to avoid metabolic stress in shrimps (Hall \& Van Hamm 1998).

The occurrence of EHP from $P$. monodon samples collected from 2 ponds, from location A \& B proven with 18S rRNA gene by PCR based diagnostic analysis. According to Artanto et al. (2020), SSU rRNA genes are used in PCR for species identification and pathogen diagnostics because the sequences usually differ between different species and similar with the same species. It is supported by Jaroenlak et al. (2016) where SSU rRNA sequence is appropriate for use within cultivated shrimp samples compared with environmental samples which have to be reconfirm using spore wall protein primers.
As per the phylogenetic analysis, the phylogenetic tree revealed that all the EHP samples were clustered based on geographical continents (Asian vs Latin America). Malaysia samples from Location A formed monophyletic clade within the EHP cluster of other Asian countries' samples. This confirms that EHP infected samples from Malaysia are genetically close with each other except for samples from Location B, which formed a new cluster by itself. It is supported with the pairwise genetic distance analysis where samples obtained from Location A genetically related with shown (0.2\% variance) with Asian countries compared with $4.9 \%$ variance in the samples identified from Location $B$. Genetic distance between Location A and Latin America is $1.7 \%$ and Location $B$ is $6.1 \%$ compared with only $1.4 \%$ with other Asian countries. Genetic distances showed that intercountry genetic variation is higher than the intra-country relationships. This divergence analysis and also the phylogenetic analysis supporting that samples from Location B could be a new species within the same family of Enterocytozoonidae. The genetic distance of Location B samples with the outgroup, E. bieneusi showing a variance of $10 \%$. According to Tourtip et al. (2009), EHP was identified based on ultrastructural features which are unique to the family Enterocytozoonidae even though SSU rRNA sequences were matching $84 \%$ similarity with the E. bieneusi. Thus, further analyses have to be done to further confirm the status of species from Location B.

Variation occurred in the sample from Latin America (KY593129). According to Artanto et al. (2020), Latin American sample have shown in a separate clade

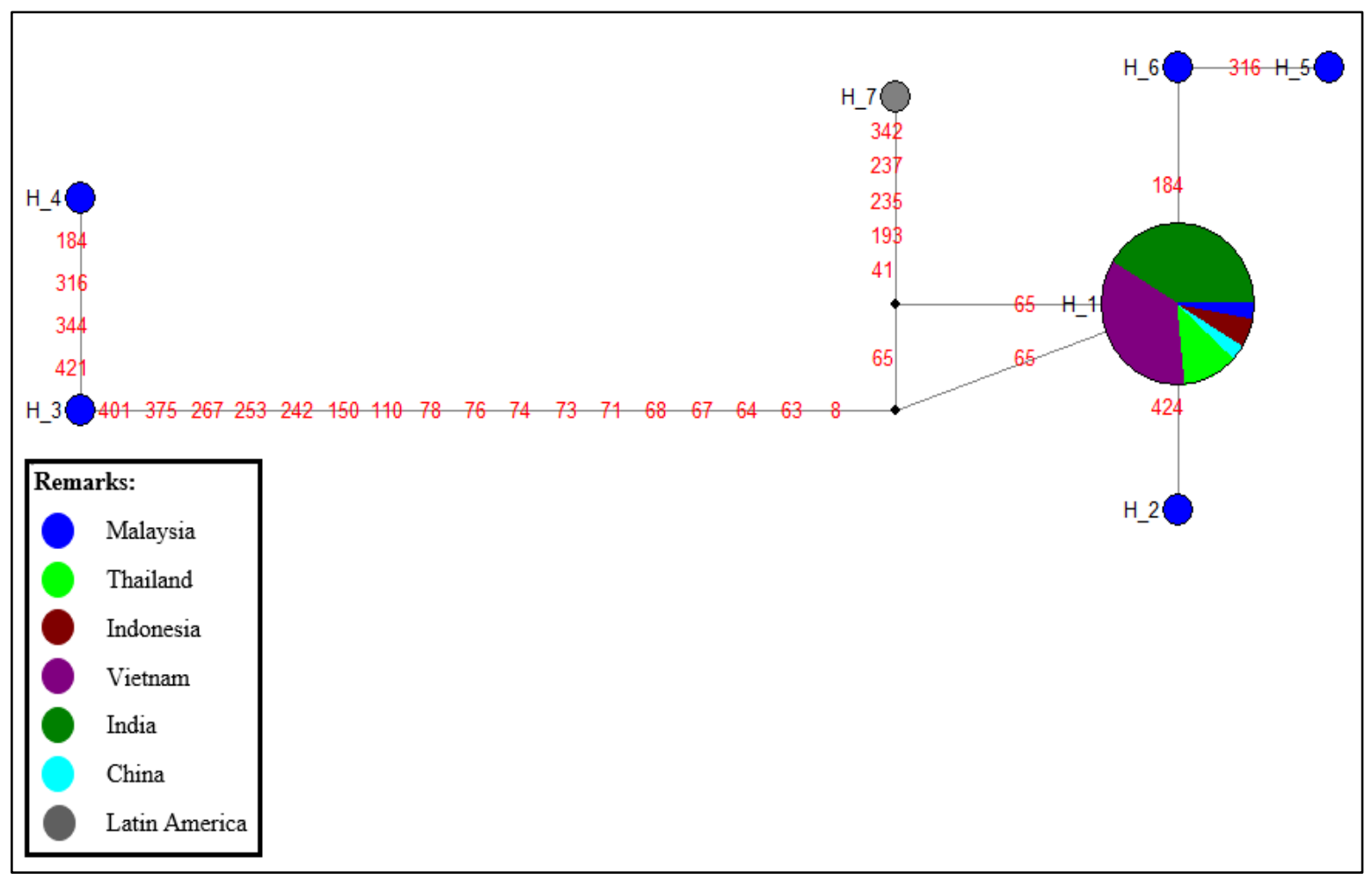

Figure 4. Minimum- spanning network of EHP from various countries haplogroup defining 7 haplotypes. Haplotype 3 and 4 are from Location B samples, Haplotype 1,2,5, and 6 are from Location A samples, and Haplotype 7 is from Latin America. 
with different branches of EHP groups from Indonesia, Thailand, Vietnam, China, and India. Chaijarasphong et al. (2020) disclosed that EHP reported from Venezuela, (KY 593127) found in the hepatopancreas of $P$. vannamei is questionable where the low similarity in gene sequences of the $\beta$-tubulin (93\%) and spore wall protein (91\%) genes to samples from Thailand (Tourtip et al., 2009) would be a different or unknown species within the early growth mortality group. Enterocytozoon-group microsporidia (EGM) has expanded rapidly, and it is now known to also contain parasites from several other genera (including Enterospora, Nucleospora, Desmozoon, Obruspora and Hepatospora). They affect a broad range of invertebrate and vertebrate hosts, including humans and inhabit diverse biomes (Stentiford et al., 2019). With this increased knowledge on diversity and the host-range comes an increasing need to carefully apply appropriate taxonomic tools when determining the presence of a pathogen of concern within a particular setting (Stentiford et al., 2014). Therefore, further analysis is a must to confirm the Location B samples.

The current clinical investigation determined the prevalence of EHP in 2 farms located in Selangor state. As the first step to mitigating the risk of EHP, it is a must to encourage the farmers to follow standard management strategies or best practices such as maintaining good water quality, lower stocking densities and use of EHP-free shrimp for stocking in shrimp farms to keep EHP under control as till today no treatment available.

\section{Conclusion}

Preliminary investigation results confirmed the EHP outbreak in Malaysia. The results have shown that Malaysia samples at Location A are genetically related to other Asian EHP samples. However, samples from Location B were not clustered within the EHP clade and shows the highest genetic variance within Malaysia and among Asian samples. Hence, further molecular analysis is needed to identify these samples. Nevertheless, the results of the current preliminary study can be used as baseline data to further investigate the epidemiology of this parasite and its role in the production loss of farmed shrimps.

\section{Ethical Statement}

The authors declare that they have no known competing financial interests or personal relationships that could have appeared to influence the work reported in this paper.

\section{Funding Information}

This work was supported by the (Terra Aqua Group, Centre for Research in Biotechnology for Agriculture (CEBAR), University of Malaya: https://cebar.um.edu.my/ under Grant (Grant no: RU004-2020)

\section{Author Contribution}

Shamala Marimuthu was involved in the experiments and results interpretation, data analysis and manuscript writing. Samudi Chandramathi and Subha Bhassu were involved in the experiment design and manuscript revision. All authors have given final approval for the manuscript publication.

\section{Conflict of Interest}

The authors declare that there is no conflict of interest.

\section{Acknowledgements}

Terra Aqua Group, Centre for Research in Biotechnology for Agriculture (CEBAR), University of Malaya.

Animal Genetics \& Genome Evolutionary Lab (Agagel), Faculty of Science, University of Malaya.

\section{References}

Anderson, I.G., Shariff, M., Nash, G. (1989). A hepatopancreatic microsporidian in pond- reared tiger shrimp, Penaeus monodon, from Malaysia. Journal of Invertebrate Pathology, 53, 278-280.

Bandelt, H.J, Forster, P., \& Röhl, A. (1999). Median-joining networks for inferring intraspecific phylogenies. Molecular Biology and Evolution, 16(1): 37-48.

Freeman, M.A., \& Sommerville, C. (2009). Desmozoon lepeophtherii n. gen., n. sp., (Microsporidia: Enterocytozoonidae) infecting the salmon louse Lepeophtheirus salmonis (Copepoda: Caligidae). Parasites \& Vectors, 2(1), 58.

Ha, N.T., Ha, D.T., Thuy, N.T., \& Lien, V.T.K., (2010). Occurrence of microsporidia Enterocytozoon hepatopenaei in white feces disease of cultured black tiger shrimp (Penaeus monodon) in Vietnam. Aquatic Animal Health \& Disease.

Han, B., Weiss, L.M. (2017). Microsporidia: obligate intracellular pathogens within the Fungal Kingdom. Microbiology Spectrum, 5.

Hou, D., Huang, Z., Zeng, S., Liu, J., Wei, D., Deng, X., Weng, S., Yan, Q., He, J. (2018). Intestinal bacterial signatures of white feces syndrome in shrimp. Applied Microbiology and Biotechnology

Hudson, D.A., Hudson, N.B., \& Pyecroft, S.B. (2001). Mortalities of Penaeus japonicus prawns associated with microsporidean infection. Australian Veterinary Journal, 79, 504-505.

Jaroenlak, P., Boakye, D.W., Vanichviriyakit, R., Williams, B.A.P., Sritunyalucksana, K.\& Itsathitphaisarn, O. (2018). Identification, characterization and heparin binding capacity of a spore-wall, virulence protein from the shrimp microsporidian, Enterocytozoon hepatopenaei (EHP). Parasites Vectors.

Jaroenlak, P., Sanguanrut, P., Williams, B.A.P., Stentiford, G.D., Flegel, T.W., Sritunyalucksana, K., \& Itsathitphaisarn, O. 
(2016). A nested PCR assay to avoid false positive detection of the microsporidian Enterocytozoon hepatopenaei (EHP) in environmental samples in shrimp farms. PLoS One 11.

Librado, P. \& Rozas.J. (2009). DnaSP v5: A software for comprehensive analysis of DNA polymorphism data. Bioinformatics, 25: 1451-1452.

Liu, Y.M., Zhang, Q., Wan, X.Y., Ma, F., \& Huang, J. (2016). Development of real-time PCR assay for detecting microsporidian Enterocytozoon hepatopenaei and the application in shrimp samples with different growth rates. Progress in Fishery Sciences, 37, 119-126

Liu, Y.-M., Qiu, L., Sheng, A.-Z., Wan, X.-Y., Cheng, D.-Y., Huang, J. (2018). Quantitative 959 detection method of Enterocytozoon hepatopenaei using TaqMan probe realtime PCR. Journal of Invertebrate Pathology, 151, 191196.

Rajendran, K.V., Shivam, S., Ezhil Praveena, P., Joseph Sahaya Rajan, J., Sathish Kumar, T., Avunje, S., Jagadeesan, V., Prasad Babu, S.V.A.N.V., Pande, A., Navaneeth Krishnan, A., Alavandi, S.V., Vijayan, K.K. (2016). Emergence of Enterocytozoon hepatopenaei (EHP) in farmed Penaeus (Litopenaeus) vannamei in India. Aquaculture, 454, 272280.

Sritunyalucksana, K., Sanguanrut, P., Salachan, P.V., Thitamadee, S., Flegel, T.W. (2015). Urgent appeal to control spread of the shrimp microsporidian parasite Enterocytozoon hepatopenaei (EHP). Network of Aquaculture Centres Asia-Pacific (NACA).

Stentiford, G.D., Feist, S.W., Stone, D.M., Peeler, E.J., Bass, D. (2014). Policy, phylogeny, and the parasite. Trends Parasitol.

Sudhir, K., Glen, S. \& Koichiro, T. (2016). MEGA7: Molecular Evolutionary Genetics Analysis Version 7.0 for Bigger Datasets. Molecular Biology and Evolution, 33(7):1870-4.

Tang, K.F.J., Han, J.E., Aranguren, L.F., White-Noble, B., Schmidt, M.M., Piamsomboon, P., Risdiana, E., Hanggono, B. (2016). Dense populations of the microsporidian Enterocytozoon hepatopenaei (EHP) in feces of Penaeus vannamei exhibiting white feces syndrome and pathways of their transmission to healthy shrimp. Journal of Invertebrate Pathology, 140, 1-7.

Tang, K.F.J., Pantoja, C.R., Redman, R.M., Han, J.E., Tran, L.H., Lightner, D.V. (2015). Development of in situ hybridization and PCR assays for the detection of Enterocytozoon hepatopenaei (EHP), a microsporidian parasite infecting penaeid shrimp. Journal of Invertebrate Pathology, 130, 37-41.

Thawatchai, C., Natthinee, M., Grant, D.S., Diva, J. A., Kwanta, T., Timothy, W.F., Kallaya, S. \& Ornchuma, I. (2020). The shrimp microsporidian Enterocytozoon hepatopenaei (EHP): Biology, pathology, diagnostics and control. Journal of Invertebrate Pathology. https://doi.org/10.1016/j.jip.2020.107458

Thitamadee, S., Prachumwat, A., Srisala, J., Jaroenlak, P., Salachan, P.V., Sritunyalucksana, 1104 K., Flegel, T.W., Itsathitphaisarn, O. (2016). Review of current disease threats for 1105 cultivated penaeid shrimp in Asia. Aquaculture, 452, 69-87.

Tourtip, S., Wongtripop, S., Stentiford, G.D., Bateman, K.S., Sriurairatana, S., Chavadej, J., Sritunyalucksana, K., Withyachumnarnkul, B. (2009). Enterocytozoon hepatopenaei sp. nov. (Microsporida: Enterocytozoonidae), a parasite of the black tiger shrimp Penaeus monodon (Decapoda: Penaeidae): Fine structure and phylogenetic relationships. Journal of Invertebrate Pathology, 102, 21-29.

Yohanes, K.A., Slamet, B.P., Sarjito, Desrina, Alfabetian, H.C.H.O. (2019) Molecular Characteristics of Indonesian Isolate Enterocytozoon hepatopenaei Based on Sequence Analysis of 18 S rRNA Genes. Akuatika, 15 (1), 93-102

Zhou, S., Wang, M., Liu, M., Jiang, K., Wang, B., Wang, L. (2020). Rapid detection of Enterocytozoon hepatopenaei in shrimp through an isothermal recombinase polymerase amplification assay. Aquaculture, 294, 734987 